\title{
Radiative capture rates at deep defects from electronic structure calculations
}

\author{
Cyrus E. Dreyer,${ }^{1,2,3}$ Audrius Alkauskas, ${ }^{4}$ John L. Lyons, ${ }^{5}$ and Chris G. Van de Walle ${ }^{1}$ \\ ${ }^{1}$ Materials Department, University of California, Santa Barbara, California 93106-5050, USA \\ ${ }^{2}$ Department of Physics and Astronomy, Stony Brook University, Stony Brook, New York 11794-3800, USA \\ ${ }^{3}$ Center for Computational Quantum Physics, Flatiron Institute, $1625^{\text {th }}$ Avenue, New York, NY 10010, USA \\ ${ }^{4}$ Center for Physical Sciences and Technology (FTMC), LT-10257 Vilnius, Lithuania \\ ${ }^{5}$ United States Naval Research Lab, Washington, DC 20375, USA
}

(Dated: August 7, 2020)

\begin{abstract}
We present a methodology to calculate radiative carrier capture coefficients at deep defects in semiconductors and insulators from first principles. Electronic structure and lattice relaxations are accurately described with hybrid density functional theory. Calculations of capture coefficients provide an additional validation of the accuracy of these functionals in dealing with localized defect states. We also discuss the validity of the Condon approximation, showing that even in the event of large lattice relaxations the approximation is accurate. We test the method on GaAs: $V_{\mathrm{Ga}}-\mathrm{Te}_{\mathrm{As}}$ and $\mathrm{GaN}: \mathrm{C}_{\mathrm{N}}$, for which reliable experiments are available, and demonstrate very good agreement with measured capture coefficients.
\end{abstract}

PACS numbers: 78.47.jd,78.55.Cr,78.55.Et,71.55.-i

\section{INTRODUCTION}

Optical techniques such as absorption, photoluminescence (PL), PL excitation spectroscopy, and timedependent PL are powerful tools for studying defects in

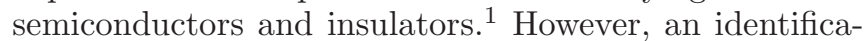
tion of the microscopic nature of the defects that give rise to specific optical signatures often requires quantummechanical calculations that address the atomic and electronic structure at the microscopic level. Specifically, predictive calculations of radiative capture rates can be compared with rates determined from time-dependent PL measurements ${ }^{2}-\underline{\underline{4}}$ to provide a microscopic identification of the defects that give rise to optical transitions.

Radiative processes may also be involved in defectmediated Shockley-Read-Hall (SRH) recombination, particularly in wide-band-gap materials. Ascertaining whether radiative recombination channels can be detrimental to device performance requires a quantitative understanding of radiative capture rates at deep defects.

In the past, calculations of carrier capture coefficients were based on analytical models ${ }^{7-10}$ Such models do not account for the complexity of the electronic structure of deep defects, which involves, e.g., strong lattice relaxations that often break the local symmetry.

In this paper we demonstrate a first-principles implementation for the calculation of radiative carrier capture rates at defects in semiconductors and insulators. We will use two well-characterized defects as case studies: a Ga vacancy and $\mathrm{Te}$ donor complex in $\mathrm{GaAs}_{2}, \frac{2}{2}$ and a carbon substitutional impurity on a nitrogen site in $\mathrm{GaN}_{2}^{3,11}$ to show that calculations based on hybrid density functionals are in excellent agreement with experimental capture coefficients. We quantify the errors resulting from key approximations and perform comparisons with model calculations. First-principles calculations of radiative capture at a carbon impurity in GaN were also reported by Zhang et $a l \stackrel{12}{=}$ In our work we present a detailed derivation of the carrier capture rate and point out differences with the work of Ref. 12 that are important for quantitative accuracy, as evidenced by comparison with experiment.

\section{FORMALISM}

\section{A. Radiative capture in semiconductors and insulators}

Radiative capture in a material with band gap $E_{g}$ is illustrated in Fig. 1(a). Let us consider a single acceptor defect, $A$, with a level in the lower part of the band gap. The optical process consists of the capture of an electron from the conduction band: $A^{0}+e^{-} \rightarrow A^{-}$. $E_{\mathrm{ZPL}}$ is the zero-phonon line, given by the position of the $(0 /-)$ charge-state transition level below the conduction-band minimum $(\mathrm{CBM})$. Let $N_{A}^{0}$ be the concentration of acceptors in the neutral charge state, and $n$ the density of electrons. The rate of the radiative process (i.e., the number of radiative events per unit time per unit volume) is given by $R_{n}=C_{n} N_{A}^{0} n$, where $C_{n}$ (units: $\mathrm{cm}^{3} \mathrm{~s}^{-1}$ ) is the radiative electron capture coefficient. A similar equations applies to radiative capture of holes, with a capture coefficient $C_{p}$. Determining $C_{n}$ and $C_{p}$ is the main goal of the present paper. Instead of coefficients $C_{\{n, p\}}$, capture cross sections $\sigma$ are often used. The two are related via $C=v \sigma$, where $v$ is the characteristic carrier velocity; for non-degenerate carriers $v$ is the thermal velocity $\underline{\underline{10}}$

The radiative transition can also be represented via a configuration coordinate diagram [Fig. 1(b)]. The two charge states of the defect give rise to curves that are displaced along the horizontal axis because generally they have different atomic configurations (here projected on a one-dimensional coordinate $Q$ ). In the so-called FranckCondon approximation, the transition occurs for fixed nuclear coordinates [see the green arrow in Fig. 1(b)] with energy $E_{\mathrm{opt}}$. After the transition the defect is in 
(a)

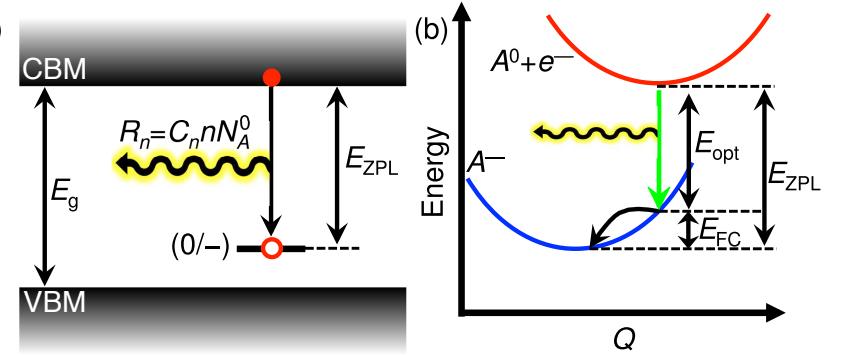

FIG. 1. Illustration of radiative carrier capture in two different representations: (a) band diagram and (b) configuration coordinate diagram, for the case of a single acceptor defect $A$.

a vibrationally excited state. This state will decay to the equilibrium state on the picosecond time scale via phonon-phonon interactions, losing the relaxation energy $E_{\mathrm{FC}}=E_{\mathrm{ZPL}}-E_{\mathrm{opt}}$, called the Franck-Condon energy.

The strength of the electron-phonon coupling associated with an optical transition can be expressed in terms of the Huang-Rhys factor $S \stackrel{13}{\underline{13}}$ which quantifies the number of phonons emitted during the transition. In this work, we will consider defects with strong electronphonon coupling $(S \gg 1)$. For such defects, $E_{\text {opt }}$ corresponds to the peak of the PL spectrum $\underline{14}$ The general formalism to treat optical transitions in semiconductors is presented in textbooks (e.g., Ch. 5 of Ref. 10 or Ch. 10 of Ref. 15). Here we will present a derivation of the capture coefficients specifically adapted to our implementation within first-principles electronic structure theory, and focusing on the case of strong electron-phonon coupling.

We will closely follow the reasoning previously applied in deriving nonradiative capture coefficients 16 [summarized in the supplemental material $(\mathrm{SM}) \frac{17}{17}$ section S1], where it was shown that, for defects in the dilute limit, the capture coefficient can be expressed as

$$
C_{n}=V r,
$$

where $r$ is the capture rate of one electron by one impurity in the volume $V$; the task is to calculate $r$.

\section{B. Derivation of the capture coefficient}

The wavefunctions describing the defect system are functions of all electronic $\{x\}$ and ionic $\{Q\}$ degrees of freedom; using the Born-Oppenheimer approximation, they can be written in the form $\Psi(\{Q\},\{x\}) \chi(\{Q\})$, where $\Psi(\{Q\},\{x\})$ is the electronic wavefunction (which depends parametrically on $\{Q\})$, and $\chi(\{Q\})$ is the ionic wavefunction. Let the electronic wavefunction of the initial (excited) state, which in the case of the acceptor in Fig. 1 is the neutral defect plus the electron in the conduction band, be $\Psi_{i}(\{Q\},\{x\})$; the associated ionic wavefunctions are $\chi_{i m}(\{Q\})$, where $m$ denotes the vibrational state. We will consider only transitions at low temperature, and therefore initially the system is in the ground vibrational state $(m=0)$. The corresponding quantities for the final (ground) electronic state (the negatively charged defect, Fig. 1) are $\Psi_{f}(\{Q\},\{x\})$ and $\chi_{f n}(\{Q\})$. The expressions can be easily generalized to finite temperatures $\frac{15}{\underline{15}}$

Optical transitions occur because of coupling to the electric field, described by the momentum matrix element $\mathbf{P}_{i f}(\{Q\})=\left\langle\Psi_{i}(\{Q\})|\hat{\mathbf{P}}| \Psi_{f}(\{Q\})\right\rangle$; the momentum operator is $\hat{\mathbf{P}}=-i \hbar \sum_{j} \partial / \partial \mathbf{x}_{j}$, where the sum runs over all electrons $j$. We will use the Condon approximation $(\mathrm{CA}), \frac{18}{\Perp}$ in which the dependence on $\{Q\}$ is neglected and the momentum matrix element is taken at a fixed $\{Q\}$ (which we choose to be the equilibrium geometry of the initial state); the validity of the CA will be discussed below.

An additional approximation is that multi-electron wavefunctions $\Psi_{\{i, f\}}$ can be replaced with single-particle Kohn-Sham orbitals $\psi_{i}$ and $\psi_{f}$, with corresponding momentum matrix element, $\mathbf{p}_{i f}$. In the case of electron capture, $\psi_{i}$ is a perturbed conduction-band state, while $\psi_{f}$ is a defect state. At finite temperature, electrons occupy a thermal distribution of states with different momenta; in principle, one has to average over this distribution. For non-degenerate semiconductors at room temperature these states are very close to the CBM, and thus we will approximate the initial state to be the CBM.

Within the Born-Oppenheimer approximation and the $\mathrm{CA}$, the luminescence intensity (number of photons per unit time, per unit energy, for a given photon energy $\hbar \omega$ ) is given by $\underline{15}$

$$
\begin{aligned}
I(\hbar \omega)= & \frac{e^{2} n_{\mathrm{r}} \omega \eta_{\mathrm{sp}}}{3 m^{2} \varepsilon_{0} \pi c^{3} \hbar}\left|p_{i f}\right|^{2} \\
& \times \sum_{n}\left|\left\langle\chi_{i 0} \mid \chi_{f n}\right\rangle\right|^{2} \delta\left(E_{\mathrm{ZPL}}-\hbar \omega_{f n}-\hbar \omega\right) .
\end{aligned}
$$

$n_{\mathrm{r}}$ is the index of refraction, $\hbar \omega_{f n}$ is the energy of the final vibrational state (with respect to its ground state), and $\eta_{\mathrm{sp}}$ is a factor which accounts for the spin selection rule $\left(\eta_{s p}=1\right.$ for a transition from a spin-singlet to a doublet, $\eta_{s p}=0.5$ for a transition from a doublet to a singlet or from a triplet to a doublet). The total recombination rate is the integral of $I(\hbar \omega)$ over energy $\hbar \omega$ :

$$
r=\frac{e^{2} n_{r} \eta_{\mathrm{sp}}}{3 m^{2} \varepsilon_{0} \pi c^{3} \hbar^{2}}\left|p_{i f}\right|^{2}\langle\hbar \omega\rangle,
$$

where $\langle\hbar \omega\rangle=\sum_{n}\left|\left\langle\chi_{i 0} \mid \chi_{f n}\right\rangle\right|^{2}\left(E_{Z P L}-\hbar \omega_{f n}\right)$ is the average energy of emitted photons. In the case of strong electron-phonon coupling, $\langle\hbar \omega\rangle$ coincides with the energy of the vertical transition $E_{\text {opt }}$ [green arrow in Fig. [1(b)]. For defects studied in this work $S>8$, so we will make this approximation.

Combining Eq. (11) and Eq. (3) gives the capture coefficient if the quantities in Eq. (3) could be calculated in a large volume $V$ corresponding to the dilute limit of defects; in practice, calculations are performed in supercells with much smaller volumes $\widetilde{V}$. The limited supercell 
size is not an issue for describing capture at neutral defects. However, in the case of charged defects the initial electronic state is not properly described. This issue can be accounted for by scaling Eq. (3) by the so-called Sommerfeld factor $f: \underline{16,19,20}$ In this work, only neutral centers are considered, so $f=1$.

The final expression for the capture coefficient is:

$$
\begin{aligned}
C_{n} & =f \eta_{\mathrm{sp}} \widetilde{V} \frac{e^{2} n_{\mathrm{r}}}{3 m^{2} \varepsilon_{0} \pi c^{3} \hbar^{2}}\left|p_{i f}\right|^{2} E_{\mathrm{opt}} \\
& =\left(5.77 \times 10^{-17}\right)\left(\widetilde{V} f n_{r} \eta_{\mathrm{sp}} E_{\mathrm{opt}} \frac{\left|p_{i f}\right|^{2}}{2 m}\right) \mathrm{cm}^{3} \mathrm{~s}^{-1}
\end{aligned}
$$

where in the second line we have evaluated the materialindependent parameters (assuming $E_{\text {opt }}$ and $\left|p_{i f}\right|^{2} / 2 m$ are expressed in $\mathrm{eV}$, and $\widetilde{V}$ in $\AA^{3}$ ) resulting in a simple formula that can be used to evaluate radiative capture coefficients based on quantities generated by density functional theory calculations. Equation (4) agrees with the expression used in Ref. 12, except for the fact that the spin selection rules are neglected in that work (e.g., for carbon on the $\mathrm{N}$ site in $\mathrm{GaN}$, this results in an extra factor of two in Ref. 12).

\section{RESULTS}

\section{A. Computational details}

We have calculated $E_{\text {opt }}$ and $\mathbf{p}_{i f}$ necessary for Eq. (4) using density functional theory with the hybrid functional of Heyd, Scuseria, and Ernzerhof (HSE) $\underline{21}$ The mixing parameter was chosen to reproduce the experimental band gaps: 0.30 for $\mathrm{GaAs}^{22}$ (giving a band gap of $1.52 \mathrm{eV}$ ) and 0.31 for GaN (giving a band gap of 3.50 $\mathrm{eV})$. Ga $3 d$ electrons were treated as core states. Defect calculations were performed on 216-atom zincblende supercells for GaAs, and 96-atom wurtzite supercells for GaN. When optimizing the defect geometry the Brillouin zone was sampled with a single special $k$-point $(1 / 4,1 / 4,1 / 4) \stackrel{23}{n}$ Since for non-degenerately doped GaN and GaAs the electrons participating in capture originate from the CBM, momentum matrix elements were evaluated at the $\Gamma$ point. Use of the $\Gamma$ point also correctly captures the symmetries of the system. We used the Vienna ab-initio Simulation Package (VASP) 24 with the projector augmented wave (PAW) method $\stackrel{25}{\rightleftharpoons}$ for the transition matrix elements, the methodology of Ref. 26 was used, (i.e., momentum matrix elements are correctly calculated for the case of nonlocal potentials). Thermodynamic charge-state transition levels of the defects ( $E_{\mathrm{ZPL}}$ in Fig. 10) and $E_{\mathrm{opt}}$ were calculated using the standard methodology described in Ref. 27, where we use the scheme of Refs. 28 and 29 to correct for interactions between charged defects and their periodic images. We use experimental indices of refraction (3.4 for $\mathrm{GaAs}^{30}$ and 2.4 for $\mathrm{GaN}_{31}^{31}$ consistently chosen for energies $\left.E_{\mathrm{opt}}\right)$.
TABLE I. Calculated (Calc.) and experimentally measured (Exp.) optical transition energies $E_{\mathrm{ZPL}}$ and $E_{\mathrm{opt}}$ and electron capture coefficients $C_{n}$ for the two defects in our case studies.

\begin{tabular}{c|cc|cc|cc}
\hline \hline & $E_{\text {ZPL }}(\mathrm{eV})$ & $E_{\text {opt }}(\mathrm{eV})$ & \multicolumn{2}{|c}{$C_{n}\left(10^{-13} \mathrm{~cm}^{3} \mathrm{~s}^{-1}\right)$} \\
& Calc. & Exp. & Calc. & Exp. & Calc. & Exp. \\
\hline GaAs: $V_{\mathrm{Ga}}-\mathrm{Te}_{\mathrm{As}}$ & 1.23 & $1.38^{\mathrm{a}}$ & 1.02 & $1.18^{\mathrm{a}}$ & 3.5 & $6.5^{\mathrm{a}}$ \\
GaN:C & 2.48 & $2.57^{\mathrm{b}}$ & 2.01 & $2.2^{\mathrm{b}}$ & 0.7 & $0.6-1.2^{\mathrm{b}}$ \\
\hline \hline
\end{tabular}

a Ref. 2

b Refs. 3 , 4

\section{B. Capture coefficients of test-case defects}

We test the methodology on two defects for which extensive experimental information is available: the complex between a Ga vacancy and a Te donor on a nearest-

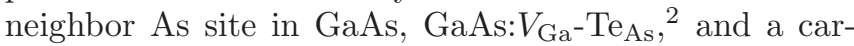
bon substitutional impurity on a nitrogen site in GaN, GaN: $\mathrm{C}_{\mathrm{N}}{ }^{3,11}$ (see Secs. S2 and S3 of SMำ for details of the experimental identification). In both cases, we examine the rate of electron capture for the neutral charge state.

We calculate the energy of the $(0 /-)$ thermodynamic charge-state transition level, $\stackrel{27}{ }$ at which electron capture occurs, to be $0.37 \mathrm{eV}$ above the VBM for GaAs: $V_{\mathrm{Ga}}-\mathrm{Te}_{\mathrm{As}}$ and $1.02 \mathrm{eV}$ for GaN: $\mathrm{C}_{\mathrm{N}}$. The calculated and experimental optical transition energies are given in Table [. A detailed description of the electronic structure of GaN: $\mathrm{C}_{\mathrm{N}}$ and GaAs: $V_{\mathrm{Ga}}-\mathrm{Te}_{\mathrm{As}}$ is provided in Sec. S4 of the SM: 17

Our calculated capture coefficients using Eq. (4) are given in Table I along with experimental values. The calculated value for $\mathrm{GaN}: \mathrm{C}_{\mathrm{N}}$ of $C_{n}=0.7 \times 10^{-13} \mathrm{~cm}^{3} \mathrm{~s}^{-1}$, is in good agreement with measurements by Reshchikov et al $\stackrel{3.4}{\text { that yield values }}(0.6-1.2) \times 10^{-13} \mathrm{~cm}^{3} \mathrm{~s}^{-1}$ for radiative capture coefficients pertaining to yellow luminescence in GaN (different values are for different samples). Therefore, our calculations indicate that $\mathrm{GaN}: \mathrm{C}_{\mathrm{N}}$ is the likely source of the yellow luminescence in the samples studied by Reshchikov $: 3$

Our value for $C_{n}$ is about a factor of four smaller than the one calculated in Ref. 12, which is mainly due to the fact that $\eta_{\mathrm{sp}}$ is not included in that work. Additionally, we find a slightly smaller value of $\left|p_{i f}\right|^{2}$ (0.03 versus 0.05 in Ref. 12), and a different value for the refractive index may have been used. We note that inclusion of $\eta_{s p}$ (i.e., application of the spin selection rules) is important and necessary to obtain agreement with experiment.

For GaAs: $V_{\mathrm{Ga}}-\mathrm{Te}_{\mathrm{As}}$, we find that $C_{n}=3.5 \times 10^{-13}$ $\mathrm{cm}^{3} \mathrm{~s}^{-1}$, five times larger than for GaN: $\mathrm{C}_{\mathrm{N}}$. Our calculated value is in satisfactory agreement with the value determined experimentally by Glinchuk et $a l^{2} \stackrel{2}{*}\left(C_{n}=\right.$ $\left.6.5 \times 10^{-13} \mathrm{~cm}^{3} \mathrm{~s}^{-1}\right)$.

In order to test convergence with supercell size, we determined $C_{n}$ for the case of $\mathrm{GaN}: \mathrm{C}_{\mathrm{N}}$ using a matrix element $\mathbf{p}_{i f}$ calculated in supercells with various sizes. A 72-atom supercell results in $C_{n}=0.29 \times 10^{13} \mathrm{~cm}^{3} \mathrm{~s}^{-1}$, while a 300-atom supercell, gives $C_{n}=0.90 \times 10^{13}$ 


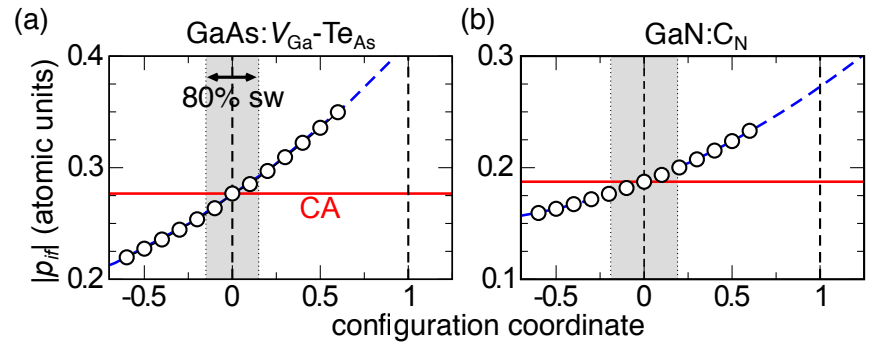

FIG. 2. Dependence of the momentum matrix elements $p_{i f}$ on configuration coordinate $Q$ for test-case defects (a) GaAs: $V_{\mathrm{Ga}^{-}}$ $\mathrm{Te}_{\mathrm{As}}$ and (b) GaN: $\mathrm{C}_{\mathrm{N}}$. The blue dashed line represents a quadratic fit to the data. The gray vertical bar indicates the spread of $Q$ values that gives rise to $80 \%$ of the spectral weight (sw). The horizontal line indicates the Condon approximation $(\mathrm{CA})$, in which the matrix element is taken to be constant.

$\mathrm{cm}^{3} \mathrm{~s}^{-1}$. This indicates that the results for the supercells used in this study are close to converged.

In Sec. S5 of the SM, 17 we compare these HSE results to those obtained using a generalized gradient functional, demonstrating the necessity of hybrid functionals for such quantitative accuracy.

\section{DISCUSSION}

\section{A. Accuracy of the Condon approximation}

As mentioned above, the derivation of Eq. (4) relies on the validity of the $\mathrm{CA}$, which states that the transition matrix element does not change with configuration coordinate. We now test this assumption for the two case studies by calculating $\left|p_{i f}\right|$ for different $Q$ values (Fig. 2). The generalized configuration coordinate chosen is a linear interpolation of all atomic positions between the ground-state structures of the neutral and negatively charged defects. This choice of $Q$ has been demonstrated to yield accurate PL lineshapes,,$\underline{14}$ indicating that it is a good approximation for the sum over all vibrational degrees of freedom.

In Fig. 2 the CA is indicated by the red horizontal line, reflecting the $\left|p_{i f}\right|$ values at $Q=0$ (the equilibrium geometry of the initial state). In order to estimate the error we make by using the CA, we must have some measure of the importance that $Q$ values other than $Q=0$ carry in a full determination of the transition rate. Such a measure is obtained by calculating the ground-state vibrational wavefunction in the initial state $\left[\left(A^{0}+e^{-}\right)\right.$in Fig. 1(b)], the square of which is roughly proportional to the spectral weight of the optical transition at a given $Q$. We then consider the variation of the matrix elements over the region containing $80 \%$ of the spectral weight of the transition (gray shaded region in Fig. 2). We see that most of the spectral weight of the transition is concentrated near the vertical transition at $Q=0$; this is gener- ally true for defects with large Huang-Rhys factors, such as the ones considered here. The matrix element $\left|p_{i f}\right|$ varies by $\sim 14 \%$ over the gray range for both defects (Fig. 22), translating into an error in $C_{n}$ of less than $14 \%$, which is acceptable and well within the experimental uncertainty. It remains to be seen if the accuracy of the CA holds for other deep defects.

\section{B. Implications for Shockley-Read-Hall recombination}

The results in Table \ provide important information about the role of radiative capture in defect-assisted $\mathrm{SRH}$ recombination processes. In Ref. 32, it was shown that for defect densities of $10^{16} \mathrm{~cm}^{-3}$, capture coefficients larger than $10^{-10} \mathrm{~cm}^{3} \mathrm{~s}^{-1}$ are necessary to result in SRH recombination rates that would compete with electronhole radiative recombination and significantly impact the performance of light-emitting diodes. If we use this magnitude as a threshold, we see that for the defects in Table I the radiative electron capture rates are much too slow (by three orders of magnitude) to give rise to detrimental SRH recombination. We suggest that this conclusion may be more general. Based on the character of the wavefunctions, we expect the optical transition matrix elements for our case-study defects to be fairly strong, and hence it seems unlikely that $\left|p_{i f}\right|^{2} / 2 m$ values for other defects (including in other hosts) would be orders of magnitude larger. Furthermore, Eq. (4) shows that $C_{n}$ depends only linearly on $E_{\mathrm{opt}}$. Both observations indicate that radiative capture coefficients are unlikely to be high enough to give rise to strong defect-assisted SRH recombination.

\section{Comparison to model calculations}

We now discuss how our methodology and implementation differ from previous attempts at theoretical descriptions of optical transitions for defects in semiconductors. Previous methods relied on models for the defect wavefunction in order to determine $\mathbf{p}_{i f}, \underline{\underline{10}}$ An often-used model for deep defects is the "quantum defect" (QD) model,$\underline{\underline{7}-\underline{9}}$ where the defect potential near the core is treated as a square well, while the long-range part has the form of a Coulomb potential. It can be shown (see Sec. S6 of the $\mathrm{SM}^{17}$ ) that, for capture of an electron at a neutral acceptor, the QD model results in a form of the capture coefficient similar to Eq. (4), but with the key difference that $\left|p_{i f}\right|^{2}$ is replaced by the momentum matrix element between the bulk conduction and valence bands $\left|p_{c v}\right|^{2}$. The matrix element is then scaled by an "effective volume" describing the spatial extent of the defect wavefunction. In addition, the QD model uses the zero-phonon line energy $\left(E_{\mathrm{ZPL}}\right)$ of the defect instead of $E_{\text {opt }}$; i.e., the Frank-Condon relaxation energy, resulting from the coupling with the lattice, is neglected. 
We now compare capture coefficients calculated with the QD model with our full first-principles results. The equations and parameters are included in Sec. S6 of the $\mathrm{SM} .17$ We find that for $\mathrm{GaN}: \mathrm{C}_{\mathrm{N}}, C_{n}^{\mathrm{QD}}=0.4 \times 10^{-13}$ $\mathrm{cm}^{3} \mathrm{~s}^{-1}$, which is smaller than our first-principles value (Table I), and slightly below the experimental range. For GaAs: $V_{\mathrm{Ga}}-\mathrm{Te}_{\mathrm{As}}, C_{n}^{\mathrm{QD}}=10.2 \times 10^{-13}$, slightly larger than the first-principles value and overestimating the experimental value. While for these case studies the model agrees reasonably well with first-principles results, it is important to emphasize the limited predictive power of models such as the QD model. First, they require energy levels taken either from experiment or from firstprinciples calculations. Second, since $\left|p_{i f}\right|$ is replaced with $\left|p_{c v}\right|$, specific information about the defect electronic structure is lost, and assumptions about the character of the defect wavefunction are required. In our case studies, the assumption that, as acceptors, the wavefunctions have valence-band character turns out to be reasonable, but this will not universally be the case.

\section{CONCLUSIONS}

We have demonstrated a methodology for determining capture coefficients from first principles. For the two case studies considered, GaAs: $V_{\mathrm{Ga}}-\mathrm{Te}_{\mathrm{As}}$ and $\mathrm{GaN}: \mathrm{C}_{\mathrm{N}}$, the calculations give quantitative agreement with experimental measurements. We also confirmed the validity of the
Condon approximation, a result that can be generalized to all defects with large values of the Huang-Rhys factor. The procedure outlined in this work will provide a tool for the identification and characterization of defects detected by optical spectroscopy, and aid in identifying the origins and mechanisms of Shockley-Read-Hall recombination.

\section{ACKNOWLEDGMENTS}

We thank D. Wickramaratne for advice on the firstprinciples calculations, and M. A. Reshchikov (VCU) for fruitful interactions and for bringing the studies of defects in GaAs to our attention. Work by C.E.D. was supported by the US Department of Energy (DOE), Office of Science, Basic Energy Sciences (BES) under Award No. de-sc0010689. Work by A.A. was supported the European Union's Horizon 2020 research and innovation programme under grant agreement No. 820394 (project ASTERIQS). J.L.L. acknowledges support from the US Office of Naval Research (ONR) through the core funding of the Naval Research Laboratory. Computational resources were provided by the National Energy Research Scientific Computing Center, which is supported by the DOE Office of Science under Contract No. DE-AC0205CH11231. The Flatiron Institute is a division of the Simons Foundation.
1 G. Davies, in Identification of Defects in Semiconductors, edited by M. Stravola (Academic Press, New York, 1999) Chap. 1: Optical Measurements of Point Defects.

2 K. D. Glinchuk, A. V. Prokhorovich, V. E. Rodionov, and V. I. Vovnenko, Phys. Status Solidi A 41, 659 (1977)

3 M. A. Reshchikov, AIP Conf. Proc. 1583, 127 (2014)

4 M. Reshchikov, J. McNamara, M. Toporkov, V. Avrutin, H. Morkoç, A. Usikov, H. Helava, and Y. Makarov, Sci. Rep. 6, 37511 (2016)

5 W. Shockley and W. T. Read, Phys. Rev. 87, 835 (1952)

${ }^{6}$ R. N. Hall, Phys. Rev. 87, 387 (1952)

7 H. B. Bebb, Phys. Rev. 185, 1116 (1969).

${ }^{8}$ H. B. Bebb and R. A. Chapman, in Proc. 3rd Photoconductivity Conf., edited by E. M. Pell (Pergamon, Oxford, 1971) p. 245.

9 G. Lucovsky, Solid State Commun. 3, 299 (1965).

10 B. K. Ridley, Quantum Processes in Semiconductors (Oxford University Press, 2013).

11 T. Ogino and M. Aoki, Jap. J. Appl. Phys. 19, 2395 (1980).

12 H.-S. Zhang, L. Shi, X.-B. Yang, Y.-J. Zhao, K. Xu, and L.-W. Wang, Adv. Opt. Mater. 5, 1700404 (2017)

13 K. Huang and A. Rhys, in Proceedings of the Royal Society of London A: Mathematical, Physical and Engineering Sciences, Vol. 204 (The Royal Society, 1950) pp. 406-423.

14 A. Alkauskas, J. L. Lyons, D. Steiauf, and C. G. Van de Walle, Phys. Rev. Lett. 109, 267401 (2012).
15 A. M. Stoneham, Theory of Defects in Solids: Electronic Structure of Defects in Insulators and Semiconductors (Oxford University Press, 2001).

16 A. Alkauskas, Q. Yan, and C. G. Van de Walle, Phys. Rev. B 90, 075202 (2014).

17 See supplemental material [URL to be inserted by publisher] for derivation and experimental determination of the radiative capture coefficient, description of the experimental identification and electronic structure of the defects, a comparison between functionals, and details about the quantum defect model calculations, which includes Refs. 33 45.

18 M. Lax, J. Chem. Phys. 20, 1752 (1952).

19 V. L. Bonch-Bruevich, Fiz. Tverd. Tella, Sbornik II , 182 (1959).

20 R. Pässler, Phys. Status Solidi B 76, 647 (1976).

21 J. Heyd, G. E. Scuseria, and M. Ernzerhof, J. Chem. Phys. 124, 219906 (2006).

22 To account for the effect of spin-orbit coupling in GaAs, the value of 0.30 for the mixing parameter is chosen to overestimate the band gap by $0.1 \mathrm{eV}$. Then we rigidly shift the VBM up in energy by $0.1 \mathrm{eV}$.

23 H. Monkhorst and J. Pack, Phys. Rev. B 13, 5188 (1976)

${ }^{24}$ G. Kresse and J. Furthmüller, Phys. Rev. B 54, 11169 (1996).

25 P. E. Blöchl, Phys. Rev. B 50, 17953 (1994). 
26 M. Gajdoš, K. Hummer, G. Kresse, J. Furthmüller, and F. Bechstedt, Phys. Rev. B 73, 045112 (2006)

27 C. Freysoldt, B. Grabowski, T. Hickel, J. Neugebauer, G. Kresse, A. Janotti, and C. G. Van de Walle, Rev. Mod. Phys. 86, 253 (2014).

28 C. Freysoldt, J. Neugebauer, and C. G. Van de Walle, Phys. Rev. Lett. 102, 016402 (2009).

29 C. Freysoldt, J. Neugebauer, and C. G. Van de Walle, Phys. Status Solidi B 248, 1067 (2011).

30 T. Skauli, P. S. Kuo, K. L. Vodopyanov, T. J. Pinguet, O. Levi, L. A. Eyres, J. S. Harris, M. M. Fejer, B. Gerard, L. Becouarn, and E. Lallier, J. Appl. Phys. 94, 6447 (2003).

31 T. Kawashima, H. Yoshikawa, S. Adachi, S. Fuke, and K. Ohtsuka, J. Appl. Phys. 82, 3528 (1997).

32 C. E. Dreyer, A. Alkauskas, J. L. Lyons, J. S. Speck, and C. G. Van de Walle, Appl. Phys. Lett. 108, 141101 (2016).

${ }^{33}$ K. Wuyts, G. Langouche, M. Van Rossum, and R. Silverans, Phys. Rev. B 45, 6297 (1992).

34 J. Gebauer, E. Weber, N. Jäger, K. Urban, and P. Ebert, Appl. Phys. Lett. 82, 2059 (2003).

${ }^{35}$ G. Baraff and M. Schlüter, Phys. Rev. Lett. 55, 1327 (1985).
36 J. L. Lyons, A. Janotti, and C. G. Van de Walle, Appl. Phys. Lett. 97, 152108 (2010).

37 C. Seager, D. Tallant, J. Yu, and W. Götz, J. Lumin. 106, $115(2004)$.

38 J. L. Lyons, A. Janotti, and C. G. Van de Walle, Phys. Rev. B 89, 035204 (2014).

39 A. Gutkin, M. Reshchikov, and V. Sedov, Semiconductors 31, 908 (1997).

40 J. P. Perdew, K. Burke, and M. Ernzerhof, Phys. Rev. Lett. 77, 3865 (1996).

41 C. E. Dreyer, A. Janotti, and C. G. Van de Walle, Appl. Phys. Lett. 102, 142105 (2013).

42 Q. Yan, E. Kioupakis, D. Jena, and C. G. Van de Walle, Phys. Rev. B 90, 121201 (2014).

43 J. P. Perdew, M. Ernzerhof, and K. Burke, J. Chem. Phys. 105, 9982 (1996).

$44 \mathrm{C}$ Adamo and V. Barone, J. Chem. Phys. 110, 6158 (1999)

45 O. Madelung, Semiconductors: Data handbook (Springer Science \& Business Media, 2012). 


\title{
Supplemental Material: Radiative capture rates at deep defects from electronic structure calculations
}

\author{
Cyrus E. Dreyer, ${ }^{1,2,3}$ Audrius Alkauskas, ${ }^{4}$ John L. Lyons, ${ }^{5}$ and Chris G. Van de Walle ${ }^{1}$ \\ ${ }^{1}$ Materials Department, University of California, Santa Barbara, California 93106-5050, USA \\ ${ }^{2}$ Department of Physics and Astronomy, Stony Brook University, Stony Brook, New York 11794-3800, USA \\ ${ }^{3}$ Center for Computational Quantum Physics, Flatiron Institute, $1625^{\text {th }}$ Avenue, New York, NY 10010, USA \\ ${ }^{4}$ Center for Physical Sciences and Technology (FTMC), LT-10257 Vilnius, Lithuania \\ ${ }^{5}$ United States Naval Research Lab, Washington, DC 20375, USA
}

(Dated: August 7, 2020)

\section{S1. RADIATIVE CAPTURE COEFFICIENT}

In this section we will derive the expression for the capture coefficient [Eq. (1) of the main text], following the reasoning of Ref. S1. For the example of electron capture at a single acceptor, let $V$ be a large volume of the material that contains $N$ electrons, their density being $n=N / V$. Let $M_{A}^{0}$ be the total number of electron-capturing defects with a density $N_{A}^{0}=M_{A}^{0} / V$. The total density of defects is $N_{A}^{0}+N_{A}^{-}=N_{A}$. If $\lambda$ is the screening length of the coulomb potential of a charged defect (or the extent of the short-range potential for a neutral defect), then the impurity potential essentially vanishes a few $\lambda$ away from the defect. We will assume that $\lambda^{3} N_{A} \ll 1$, which means that the region where the potential of impurities is not negligible is small compared to the overall volume. This allows us to assume that the average electron density is equal to the electron density in the region of negligible impurity potential. Let us also assume that the electron density is low enough $\left(n \ll \lambda^{-3}\right)$ so that one electron interacts with only one impurity at a time (i.e., the probability of finding two electrons in the volume $\lambda^{3}$ is negligible).

Let $r$ be the capture rate of one electron by one impurity in the volume $V$. The capture rate of $N$ electrons at $M_{A}^{0}$ defects will thus be $\gamma_{n}=r M_{A}^{0} N$, which we can rewrite as

$$
\gamma_{n} / V=(V r)\left(M_{A}^{0} / V\right)(N / V)=(V r) N_{A}^{0} n .
$$

As we state in the main text, the rate of the radiative process (i.e., the number of radiative events per unit time per unit volume) is given by $R_{n}=C_{n} N_{A}^{0} n$, where $C_{n}$ is the radiative electron capture coefficient. By comparing this expression with Eq. (S1) and noting that $\gamma_{n} / V=R_{n}$, we get:

$$
C_{n}=V r
$$

where $r$ is the capture rate of one electron by one impurity in the volume $V$.

\section{S2. EXPERIMENTAL DETERMINATION OF CAPTURE COEFFICIENTS}

Experimentally, values for the radiative capture coefficient can be determined from time-dependent PL measurements under well controlled conditions $[\mathrm{S} 2$ S5]. Let us consider an $n$-type semiconductor (with electron concentration $n$ ) containing the deep acceptor as depicted in Fig. 1 of the main text. The ground state of the defect is the -1 charge state, since the Fermi level is above the $(0 /-)$ charge-state transition level. Thus, in order to capture an electron radiatively, the defect has to capture a hole first. Let us first assume that hole capture, which occurs nonradiatively, is a much faster process than radiative capture. In this case photo-generated holes will be quickly trapped at defects, and the time dependence of the luminescence will be determined by the rate at which neutral acceptor defects are re-filled with electrons: $r=C_{n} n$ (units: $\mathrm{s}^{-1}$ ). The radiative lifetime $\tau=1 / r$ is therefore:

$$
\tau=\frac{1}{C_{n} n} .
$$

$\tau$ is independent of the defect concentration [S3 S5]. The electron density $n$ can be measured independently, and thus time-dependent PL measurements provide a direct measurement of capture coefficients $C_{n}$. The error bar in the experimental determination of $C_{n}$ stems mainly from inaccuracies determining $n[\mathrm{~S} 3]$.

In more general situations $C_{n}$ is extracted from the temperature dependence of radiative decay rates, as recently described by Reshchikov et al. [S5]. In most cases these decay rates follow closely the behavior deduced from a rate-equation model, which yields an accurate determination of $C_{n}$. 


\section{S3. EXPERIMENTAL IDENTIFICATION OF THE DEFECTS IN THE CASE STUDIES}

\section{A. GaAs: $V_{\mathrm{Ga}}-\mathrm{Te}_{\mathrm{As}}$}

GaAs defect luminescence lines occurring around $1.2 \mathrm{eV}$ have long been associated with a gallium vacancy bound to a donor (either $\mathrm{S}, \mathrm{Se}$, and $\mathrm{Te}$ on the $\mathrm{As}$ site, or $\mathrm{C}, \mathrm{Si}, \mathrm{Ge}$, and $\mathrm{Sn}$ on the Ga site) [S6]. The involvement of $V_{\mathrm{Ga}}$ was postulated based on the observation that the lines were absent in GaAs samples grown from a gallium solution (gallium-rich conditions) [S7]. The lines were also absent in heavily Cd- or Zn-doped samples, which was attributed to the fact that these acceptors occupy Ga sites [S6]; in fact, this reduction was more likely due to the lowering of the Fermi level, which increases the formation energy of $V_{\mathrm{Ga}}$ and hence lowers the $V_{\mathrm{Ga}}$ concentration. In addition, the intensity of the emission increased with increasing donor concentration, again supporting the involvement of $V_{\mathrm{Ga}}$ [S6], since the formation energy of $V_{\mathrm{Ga}}$ decreases as the Fermi level moves up.

The involvement of donor species in the complex was first suggested by Williams [S6] who noted a small shift of the emission line depending on the type of the donor. For Te donors, luminescence occurs at $1.18 \mathrm{eV}$. Subsequently, it was almost universally accepted that the $1.18 \mathrm{eV}$ line in Te-doped GaAs is due to the $V_{\mathrm{Ga}}-\mathrm{Te}_{\mathrm{As}}$ complex [S2, S8 [S11]. This complex in Te-doped GaAs has been proposed to be responsible for the compensation of $\mathrm{Te}_{\mathrm{As}}$ donors in GaAs [S12, [S13]. A schematic of the structure of the $V_{\mathrm{Ga}}-\mathrm{Te}_{\mathrm{As}}$ complex is shown in Fig. [S1.

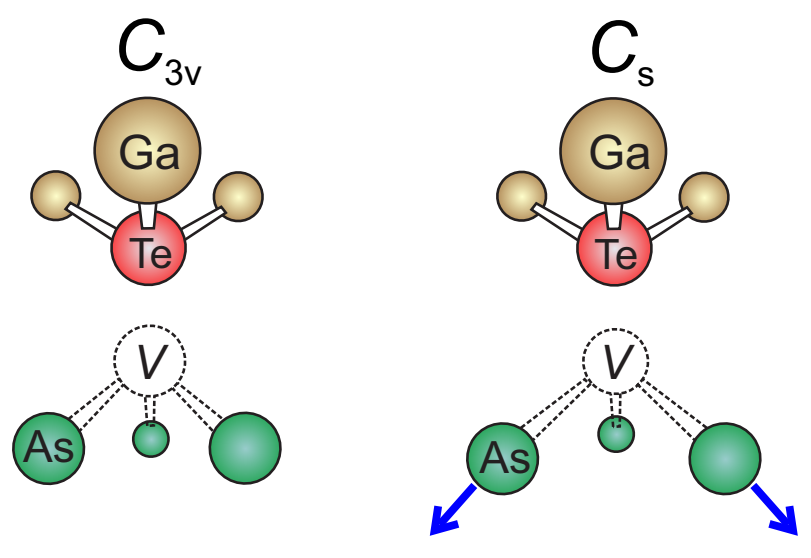

FIG. S1. Schematic of the GaAs: $V_{\mathrm{Ga}}-\mathrm{Te}_{\mathrm{As}}$ defect, in the undistorted ( $C_{3 v}$ symmetry) and distorted $\left(C_{s}\right.$ symmetry) structures. In the distorted structure, two As atoms move away from the Ga vacancy (blue arrows) and the remaining one moves closer.

Based on a careful investigation of the $1.18 \mathrm{eV}$ line in GaAs for different $n$-type doping conditions, Glinchuk et al. [S2] proposed that the luminescence line corresponds to the $(0 /-)$ charge-state transition. For donor concentrations $n>10^{17} \mathrm{~cm}^{-3}$ optical emission was caused by capture of free electrons, as evidenced from the almost linear increase of capture rates $r=C_{n} n$ and corresponding decrease of optical lifetimes according to Eq. (S3). An electron capture coefficient $C_{n}=6.5 \times 10^{-13} \mathrm{~cm}^{3} \mathrm{~s}^{-1}$ was deduced in that work [S2].

Although the attribution of the $1.18 \mathrm{eV}$ line to the $V_{\mathrm{Ga}}-\mathrm{Te}_{\mathrm{As}}$ defect seemed firm, the above conclusions about the mechanism of luminescence were challenged by the theoretical work of Baraff and Schlüter [S14]: using the Green's function implementation of DFT in the local density approximation (LDA), they found that an isolated $V_{\mathrm{Ga}}$ can occur in four charge states: $0,-1,-2$, and -3 . This suggests that the $V_{\mathrm{Ga}^{-}}-\mathrm{Te}_{\mathrm{As}}$ complex may have more charge states than just 0 and -1 assumed by Glinchuk et al. [S2]. This conclusion is confirmed by our first-principles calculations based on the HSE hybrid functional: $V_{\mathrm{Ga}}-\mathrm{Te}_{\mathrm{As}}$ indeed has three stable charge states $(0,-$, and $2-$, and thus two charge-state transition levels in the band gap: $(0 /-)$ at 1.23 below the CBM, and $(-/ 2-)$ at $1.03 \mathrm{eV}$ below the CBM. Our calculated optical transition energies $E_{\text {opt }}$ [based on a configuration coordinate diagram as illustrated in Fig. 1(b) of the main text] are $1.01 \mathrm{eV}$ for the $(0 /-)$ transition and 0.89 for $(-/ 2-)$. The result for the $(0 /-)$ transition is within $0.2 \mathrm{eV}$ of the experimental value of $1.18 \mathrm{eV}$, within the error bar that has been empirically observed for other defects when calculated with hybrid density functional theory [S15, [S16]. Thus, within the limitations of the theoretical approach, calculations support the assignment of the $1.18 \mathrm{eV}$ band to a $A^{0}+e^{-} \rightarrow A^{-}$transition.

In $n$-type material the stable charge state of the defect is $2-$. For a transition $A^{0}+e^{-} \rightarrow A^{-}$to occur, the defect must capture two holes first. This should be reflected in the defect emission being quadratically dependent on photoexcitation intensity for small intensities [S3]. However, in the case of the $1.18 \mathrm{eV}$ band this has not been observed. The reason for this is likely that nonradiative capture of holes suppresses the $A^{-}+e^{-} \rightarrow A^{-2}$ transition at even smaller photo-excited carrier densities than used in experiments of Refs. $\mathrm{S2}$, S8, S10, and $\mathbf{S 1 1}$. This is because radiative capture of an electron into the - charge state is reduced due to coulomb repulsion, and thus an extremely small hole 
concentration (and therefore extremely low excitation power) is required so that the defect in the - charge state does not rapidly transition to the neutral charge state by nonradiatively capturing a hole. When this "saturation" occurs, the intensity of the $A^{0}+e^{-} \rightarrow A^{-}$transition depends linearly on the photo-excited carrier density. Therefore, we conclude that $A^{0}+e^{-} \rightarrow A^{-}$is responsible for the observed $1.18 \mathrm{eV}$ line.

\section{B. $\mathrm{GaN}: \mathrm{C}_{\mathrm{N}}$}

Carbon is a common unintentional impurity in group-III nitride materials. In some cases it is also introduced intentionally to produce semi-insulating material. It has been shown to be one of the sources of the notorious yellow luminescence (YL) in GaN [S15, S17, S18]. Recent calculations [S15, S19] have demonstrated that GaN: $\mathrm{C}_{\mathrm{N}}$ is a deep acceptor with emission energy around $2.14 \mathrm{eV}$, in good agreement with the $2.2 \mathrm{eV}$ peak observed in experiment [S17, S18]. YL occurs when the electron in the conduction band (or at a shallow donor) is captured by a neutral carbon, $\mathrm{C}_{\mathrm{N}}^{0}$. We have previously reported first-principles calculations of the luminescence lineshape pertaining to this transition [S16]; the calculated lineshape, as well as effective vibrational parameters that characterize this lineshape, are in an excellent agreement with experiment. In addition, the nonradiative hole capture by a negatively charged carbon, $\mathrm{C}_{\mathrm{N}}^{-}$, has been calculated [S1]. In $n$-type samples this process must precede the radiative capture of electrons. Our calculated nonradiative capture coefficient $C_{p}$ was in very good agreement with experimental data by Reshchikov [S4]. Thus, in the case of $\mathrm{GaN}: \mathrm{C}_{\mathrm{N}}$, theory and experiment agree with each other regarding: (i) the energy of the radiative transition; (ii) the luminescence lineshape; (iii) nonradiative capture coefficient $C_{p}$. All of these factors reinforce the attribution of the observed $\mathrm{YL}$ in C-containing samples to $\mathrm{C}_{\mathrm{N}}$.

\section{S4. ELECTRONIC AND ATOMIC STRUCTURE OF THE DEFECTS}

\section{A. GaAs: $V_{\mathrm{Ga}}-\mathrm{Te}_{\mathrm{As}}$}

We start by considering the neutral charge state of a $V_{\mathrm{Ga}}-\mathrm{Te}_{\mathrm{As}}$ complex. First, we fix all atoms in unperturbed bulk positions. The defect then has a $C_{3 v}$ point-group symmetry, where the threefold symmetry axis goes through the site of the vacancy and the neighboring $\mathrm{Te}_{\mathrm{As}}$ atom (Fig. [S1). In this undistorted configuration there are two degenerate single-particle states of $e$ symmetry in the band gap. In the neutral charge state these are filled with two electrons. As per Hund's rule the triplet state ${ }^{3} A_{2}$ is the ground state of the defect. Let us label multi-electron states with the occupation of single-particle states $\left|e_{x} \bar{e}_{x} e_{y} \bar{e}_{y}\right\rangle$, where "bar" denotes spin-down electrons. The triplet has three components: $|1010\rangle,(|1001\rangle+|0110\rangle) / \sqrt{2}$, and $|0101\rangle$, corresponding to spin projections $m_{s}=+1,0$, and -1 .

When we allow the geometry to relax, the symmetry is lowered to $C_{s}$. The distortion decreases one $V_{\mathrm{Ga}}-\mathrm{As}$ distance and increases two others (Fig. [S1). The symmetry plane of the defect passes through the site of the vacancy, the Te atom, and the As atom that has moved inward. The single-particle $e$ states split into $a^{\prime}$ and $a^{\prime \prime}$ components. In the $C_{s}$ point group multi-electron states are given by specifying the occupations of single-particle states $\left|a^{\prime} \overline{a^{\prime}} a^{\prime \prime} \bar{a}^{\prime \prime}\right\rangle$. Like before, the triplet has three components, which can be explicitly written as $|1010\rangle,(|1001\rangle+|0110\rangle) / \sqrt{2}$, and $|0101\rangle$. The ground state of the defect in its neutral state is ${ }^{3} A^{\prime \prime}$. Note that the defect possesses three local minima, since the distortion can occur in three different directions. This is not a Jahn-Teller distortion, as assumed in Refs. S10, S20], since the electronic ground state is not degenerate $\left({ }^{3} A_{2}\right)$ in the undistorted symmetric configuration. Rather, the lowering of the symmetry of the defect should be considered polaronic, since the driving force is not the breaking of an electronic degeneracy, but rather the lowering of the electronic energy due to partial localization of electronic wavefunctions.

Next we consider the negative charge state. If we consider the undistorted configuration of the defect again, the ground state is an orbital doublet ${ }^{2} E$ with four electronic states: $|0111\rangle,|1011\rangle,|1101\rangle$, and $|1110\rangle$. Because of the electronic degeneracy, the system is unstable with respect to a Jahn-Teller distortion, and the symmetry is lowered to $C_{s}$. The electronic level splits into ${ }^{2} A^{\prime}$ and ${ }^{2} A^{\prime \prime}$. We find that the ${ }^{2} A^{\prime \prime}$ is the ground state with components $\left|a^{\prime}(1) \bar{a}^{\prime}(1) a^{\prime \prime}(1) \bar{a}^{\prime \prime}(0)\right\rangle$ and $\left|a^{\prime}(1) \bar{a}^{\prime}(1) a^{\prime \prime}(0) \bar{a}^{\prime \prime}(1)\right\rangle$. As in the case of the neutral defect, we find that in the distorted configuration one of As atoms moves towards the vacancy, while the other two move outward. Thus, the negatively charged defect possesses three local minima, each of which has exactly the same symmetry plane as the corresponding neutral defect; starting from the ground-state geometry of the neutral defect and adding one electron to the system, we end up with the defect in the negative charge state having the same symmetry plane.

From the above analysis it follows that the ground state of the defect in the neutral charge state is ${ }^{3} A^{\prime \prime}$, while that in the negatively charge state is ${ }^{2} A^{\prime \prime}$. In an optical transition an electron in the conduction band is captured into the defect state $a^{\prime}$, which is symmetric with respect to the reflection in the symmetry plane. The ground-state geometries of the two charge states are different, but both have $C_{s}$ symmetry with the same symmetry axis, and 
thus the distortion includes only fully-symmetric $a^{\prime}$ phonons. The latter result is a prerequisite to the use of the Franck-Condon approximation in optical transitions.

\section{B. $\mathrm{GaN}: \mathrm{C}_{\mathrm{N}}$}

When a nitrogen atom is replaced by a carbon atom in the undistorted GaN lattice, defect levels appear in the band gap. In the zinc-blende structure there would be three degenerate $t_{2}$ levels made of carbon $2 p$ orbitals (with an admixture of $\mathrm{N} 2 p$ orbitals on the next-nearest neighbors). In the wurtzite structure the symmetry is lowered from $T_{d}$ (tetrahedral) to $C_{3 v}$. The $t_{2}$ orbitals are split into $a_{1}$ and two degenerate $e$ orbitals.

In the negative charge state the three orbitals are filled with 6 electrons and the electronic configuration is $a_{1}^{2} e^{4}$. When we allow the geometry to relax, the negative charge state retains the $C_{3 v}$ symmetry, and the total wavefunction is of ${ }^{1} A_{1}$ symmetry. In the neutral charge state, there are 5 electrons to fill the defect states in the band gap. The ordering of these $a_{1}$ and $e$ levels is not known a priori, but has to be determined from calculations. Depending on the ordering of the levels, the ground state of $\mathrm{C}_{\mathrm{N}}^{0}$ in the undistorted $C_{3 v}$ geometry is either ${ }^{2} E$ (configuration $a_{1}^{2} e^{3}$ ) or ${ }^{2} A_{1}$ (configuration $e^{4} a_{1}$ ). These two multi-electron states are close in energy, thus, strictly speaking, $\mathrm{C}_{\mathrm{N}}^{0}$ should be classified as a Jahn-Teller (if ${ }^{2} E$ is lower in energy) or pseudo-Jahn-Teller system (if ${ }^{2} A_{1}$ is lower in energy). From the electronic structure point of view, the system is close to the triply-degenerate ${ }^{2} T_{2}$ Jahn-Teller system of the tetrahedral symmetry.

When we allow the geometry to relax, we find that the system can relax into four local energy minima. One of them corresponds to the $C_{3 v}$ symmetry, whereby the axial C-N bond is significantly elongated (2.11 $\AA$ ), while the other three retain a bond-length very similar to that of the bulk Ga-N bond length (1.96 $\AA$ ). The electronic state is of ${ }^{2} A_{1}$ symmetry. In the other three cases the symmetry is lowered to $C_{s}$, whereby one of the azimuthal C-N bonds is elongated, while the other three remain close to bulk bond lengths. The ground state in each of those configurations is of ${ }^{2} A^{\prime \prime}$ symmetry.

We find that ${ }^{2} A_{1}$ and ${ }^{2} A^{\prime \prime}$ minima are nearly-degenerate. Unfortunately, we find that the exact ordering of these states depends very sensitively on various computational parameters and cannot be determined accurately without the use of very large supercells. For modest-size supercells the distance between defects in the $x y$ plane is different from the distance in the $z$ direction, which causes an additional splitting between $a_{1}$ and $e$ orbitals beyond the natural crystal field of the wurtzite lattice. However, the calculations consistently indicate that the energy barrier between the minima is at least $0.1 \mathrm{eV}$. We thus expect this system to behave as a static Jahn-Teller system, so the Franck-Condon approximation and Born-Oppenheimer approximation apply to this defect, and we may use Eq. (4) of the main text to determine the capture coefficient. We choose the ${ }^{2} A_{1}$ structure for determining the momentum matrix element, though $\left|p_{i f}\right|$ for ${ }^{2} A^{\prime \prime}$ differs by less than $20 \%$.

\section{S5. COMPARISON BETWEEN HYBRID AND SEMILOCAL FUNCTIONALS}

In this section we comment on the role of hybrid functionals within density functional theory in providing quantitative accuracy for the capture coefficient $C_{n}$. When using traditional functionals, such as the generalized gradient approximation (GGA), the band gap is severely underestimated, and therefore the charge-state transition levels (as well as $\left.E_{\mathrm{opt}}\right)$ are also underestimated. In fact, for GaAs calculations with the Perdew, Burke, and Ernzerhof (PBE) GGA functional S21] result in a band gap of less than $0.2 \mathrm{eV}$, and therefore determining $E_{\text {opt }}$ is not possible since the transition levels do not fall within the gap. Also, because of more severe self-interaction errors in PBE, the defect wavefunctions are more delocalized than when calculated with the hybrid functional of Heyd, Scuseria, and Ernzerhof (HSE) [S22], leading to an overestimation of momentum matrix elements. HSE has also been shown to accurately capture the conduction-band dispersion compared to many-body perturbation theory techniques S23, S24].

For GaN: $\mathrm{C}_{\mathrm{N}}$, a fortuitous cancellation of these two errors occurs. Thus, the calculated $C_{n}^{\mathrm{PBE}}=0.8 \times 10^{-13} \mathrm{~cm}^{3} \mathrm{~s}^{-1}$ is reasonably close to the $\mathrm{HSE}$ value, even though $E_{\mathrm{opt}}^{\mathrm{PBE}}=0.95 \mathrm{eV}$ is severely underestimated. An important advantage of the hybrid functional approach is that it provides reliable first-principles predictions of both optical transition levels and capture coefficients, which is a crucial requirement for defect identification.

We have also performed a test with another hybrid functional, namely PBE0 [S25, S26]. We tuned the mixing parameter to 0.20 to reproduce the experimental band gap of GaN. The resulting momentum matrix element is very similar to the value obtained with $\operatorname{HSE}$ ( $8 \%$ larger). 


\section{S6. QUANTUM DEFECT MODEL FOR CALCULATING CAPTURE RATES}

Here we will compare the expression that we obtain for the radiative capture coefficient [Eq. (4) of the main text] with that obtained from the "quantum defect" (QD) model [S27, S28] that has been used previously, as reviewed in Chapter 5 of Ref. S29. In the QD model, the defect potential near the core is treated as a square well, while the long-range part has the form of a Coulomb potential. The defect wavefunction is assumed to be derived from valence-band states if it is an acceptor, and conduction-band states for donors. It is important to note that this happens to be a good approximation for the case-study defects in this work, but will likely be a poor approximation in many other cases, for instance if the wavefunction of an acceptor defect has conduction-band character.

Quantities such as the effective Rydberg energy $\left(R_{0}^{*}\right)$ and effective Bohr radius $\left(a_{0}^{*}\right)$ used below are characterized by the valence-band effective mass $m_{v}^{*}$; of course, the relevant effective mass for the density of states from which the electron originates is that of the conduction band $m_{c}^{*}$.

As in the main text, we will consider capture of an electron from the conduction band into an acceptor state, illustrated in Fig. S2. The conduction and valence bands are assumed to be parabolic. The acceptor level has an energy $E_{i}$ above the valence-band maximum $(\mathrm{VBM})$ and $E_{\mathrm{ZPL}}=E_{g}-E_{i}$ below the conduction-band minimum (CBM), where $E_{g}$ is the band gap. (cf. Fig. 1 in the main text). In the QD model, $E_{i}$ is referred to as the ionization energy. The photoionization cross section for the process $A^{-} \rightarrow A^{0}+e^{-}$is given by [Eq. (5.91) of Ref. S29]

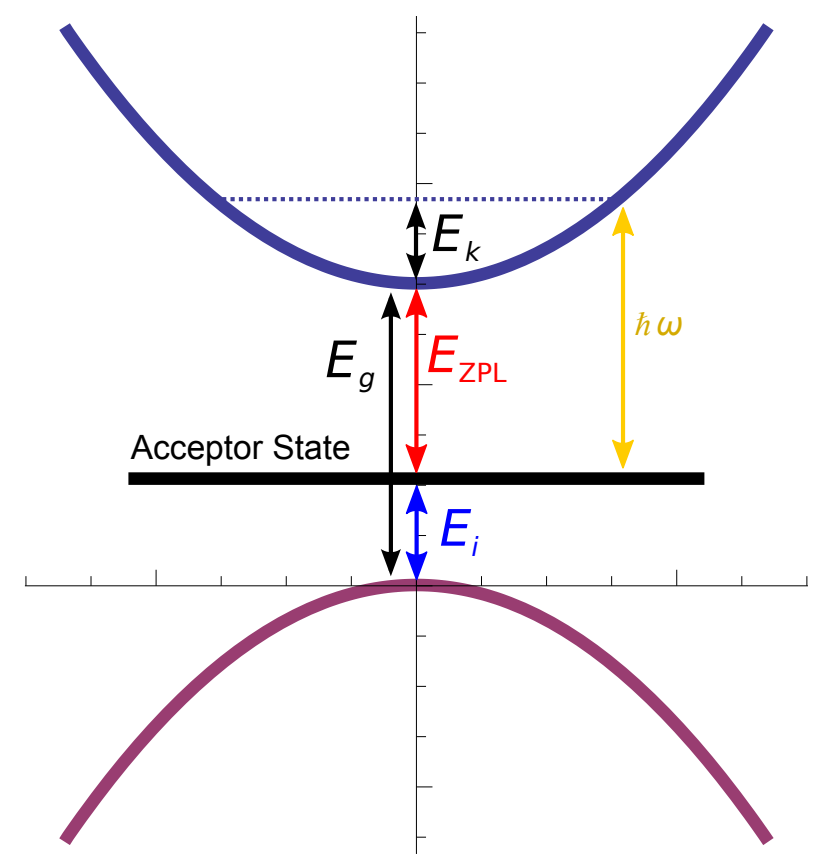

FIG. S2. Schematic of the energies involved in calculating the capture process based on the quantum-defect model.

$$
\sigma^{\mathrm{QD}}(\hbar \omega)=\frac{16 \times 2^{2 \mu}}{3} \alpha a_{0}^{2} \frac{R_{0}}{\hbar \omega} \frac{\left|p_{c v}\right|^{2}}{2 m} \frac{1}{n_{r}} \frac{2 \pi\left(\nu_{\mathrm{T}} a_{0}^{*}\right)^{3} \Gamma^{2}(\mu+1)}{\Gamma(2 \mu+1)} \frac{\left(\sin \left[(\mu+1) \tan ^{-1}\left(E_{\mathbf{k}} / E_{i}\right)^{1 / 2}\right]\right)^{2}}{\left(E_{\mathbf{k}} / E_{i}\right)\left[1+\left(E_{\mathbf{k}} / E_{i}\right)\right]^{\mu+1}}\left(\frac{2 m_{c}^{*}}{\hbar^{2}}\right)^{3 / 2} E_{\mathbf{k}}^{1 / 2},
$$

where $\alpha$ is the fine structure constant, $a_{0}$ is the Bohr radius, $R_{0}$ is the Rydberg energy, $n_{r}$ is the refractive index, $\left|p_{c v}\right|^{2}$ is the magnitude squared of the momentum matrix element between the VBM and CBM, $m$ is the free electron mass, $\hbar \omega$ and $E_{\mathbf{k}}$ are defined in Fig. S2, $\nu_{\mathrm{T}}=\sqrt{R_{0}^{*} / E_{i}}$, and $\mu=Z \nu_{\mathrm{T}}$, where $Z$ is the charge of the defect. [Note that in Eq. (5.91) of Ref. [S29, the sine term is not squared, which we attribute to a misprint in that work]. $p_{c v}$ should be distinguished from $p_{i f}$ in the main text, which is the matrix element between the CBM and the defect state; see below for discussion.

For a neutral defect $(Z=\mu=0)$, which is the relevant case for capture of electrons at the neutral deep acceptors we consider in this work, the photoionization cross section is

$$
\sigma_{Z=\mu=0}^{\mathrm{QD}}(\hbar \omega)=\frac{16}{3} \alpha a_{0}^{2} \frac{R_{0}}{\hbar \omega} \frac{1}{n_{r}} \frac{\left|p_{c v}\right|^{2}}{2 m} \frac{2 \pi\left(\nu_{\mathrm{T}} a_{0}^{*}\right)^{3}}{\left(1+E_{\mathbf{k}} / E_{\mathrm{ZPL}}\right)^{2}}\left(\frac{2 m_{c}^{*}}{\hbar^{2}}\right)^{3 / 2} E_{\mathbf{k}}^{1 / 2}
$$


We can convert Eq. (S5) into a capture coefficient by replacing the density of electron states with photon states and the photon group velocity with the electron band velocity (thus obtaining the capture cross section), and finally multiplying by the band velocity to obtain [S29]:

$$
C_{n}^{\mathrm{QD}}(\hbar \omega)=\frac{16}{3} \alpha a_{0}^{2} \frac{R_{0}}{\hbar \omega} \frac{\eta_{\mathrm{sp}}}{n_{r}} \frac{\left|p_{c v}\right|^{2}}{2 m} \frac{2 \pi\left(\nu_{\mathrm{T}} a_{0}^{*}\right)^{3}}{\left(1+E_{\mathbf{k}} / E_{\mathrm{ZPL}}\right)^{2}}\left(\frac{2 m_{c}^{*}}{\hbar^{2}}\right)^{3 / 2} E_{\mathbf{k}}^{1 / 2} \times \frac{(\hbar \omega)^{2}}{2 m_{c}^{*} E_{\mathbf{k}}\left(c / n_{r}\right)^{2}} \times \sqrt{\frac{2 E_{\mathbf{k}}}{m_{c}^{*}}} .
$$

where we have also included the factor that accounts for the spin selection rules $\eta_{\mathrm{sp}}\left(\eta_{\mathrm{sp}}=0.5\right.$ for our test cases). We consider the capture coefficient for an electron originating from the CBM (corresponding to the threshold for photoionization), i.e., $\hbar \omega=E_{\mathrm{ZPL}}$ and $E_{\mathbf{k}}=\hbar \omega-E_{\mathrm{ZPL}}=0$ :

$$
\begin{aligned}
C_{n}^{\mathrm{QD}} & =8 n_{r} \eta_{\mathrm{sp}} \frac{c}{3 a_{0}\left(m c^{2}\right)^{2}} \frac{\left|p_{c v}\right|^{2}}{2 m} E_{\mathrm{ZPL}}\left[\pi\left(\nu_{\mathrm{T}} a_{0}^{*}\right)^{3}\right] \\
& =\frac{\eta_{\mathrm{sp}} e^{2} n_{r}}{3 m^{2} \varepsilon_{0} \pi c^{3} \hbar^{2}}\left|p_{c v}\right|^{2} E_{\mathrm{ZPL}}\left[4 \pi\left(\nu_{\mathrm{T}} a_{0}^{*}\right)^{3}\right],
\end{aligned}
$$

Comparing this expression to Eq. (4) of the main text highlights two key approximations in the QD model. First, the model does not account for the coupling of the defect with the lattice, since the energy of the transition is taken to be $E_{\mathrm{ZPL}}$ (instead of $E_{\mathrm{opt}}$ ) which does not include the Frank-Condon relaxation energy. The second key approximation of the QD model is that the momentum matrix element between the conduction band and defect state is taken to be the one between the conduction and valence bands; in place of $\widetilde{V}$ of Eq. (4) of the main text (the supercell volume in which $\mathbf{p}_{i f}$ is calculated), $\left|p_{c v}\right|^{2}$ is multiplied by an effective volume of the defect $V_{\text {eff }}=4 \pi\left(\nu_{\mathrm{T}} a_{0}^{*}\right)^{3}$.

We quantitatively compare Eq. (S8) to our first-principles results using the parameters for our defects and host materials listed in Table I. We see that the QD model predicts a capture coefficient for GaAs: $V_{\mathrm{Ga}}-\mathrm{Te}_{\mathrm{As}}$ that is overestimated compared to experiment, whereas the capture coefficient for $\mathrm{GaN}: \mathrm{C}_{\mathrm{N}}$ is smaller than the experimental range.

\begin{tabular}{|c|c|c|c|c|c|c|c|c|c|c|}
\hline & $E_{\mathrm{ZPL}}(\mathrm{eV})^{\mathrm{a}}$ & $m_{c}^{*}\left(\mathrm{~m}_{\mathrm{e}}\right)^{\mathrm{b}}$ & $m_{\mathrm{h}}^{*}\left(m_{\mathrm{e}}\right)^{\mathrm{b}}$ & $\varepsilon_{r_{\text {点 }}^{\mathrm{b}}}$ & $n_{r}$ & $\left|p_{c v}\right|^{2} / 2 m(\mathrm{eV})$ & $R_{0}^{*}(\mathrm{eV})$ & $a_{0}^{*}(\AA)$ & $V_{\text {eff }}\left(\AA^{3}\right)$ & $\mathrm{C}_{n}^{\mathrm{QD}}\left(\mathrm{cm}^{3} / \mathrm{s}\right)$ \\
\hline GaAs: $V_{\mathrm{Ga}}-\mathrm{Te}_{\mathrm{As}}$ & 1.23 & 0.067 & 0.5 & 12.9 & $3.4^{\mathrm{c}}$ & $6^{\mathrm{a}}$ & 0.04 & 13.7 & 381 & $10.15 \times 10^{-13}$ \\
\hline $\mathrm{GaN}: \mathrm{C}_{\mathrm{N}}$ & 2.48 & 0.20 & 1.0 & 10.4 & $2.4^{\mathrm{d}}$ & $4^{\mathrm{e}}$ & 0.13 & 5.5 & 66 & $0.39 \times 10^{-13}$ \\
\hline
\end{tabular}

TABLE I. Parameters for defects and host materials used in Eq. ( $[\mathbf{S 8}$ ), and the resulting effective defect volume and electronic capture coefficient.

a This work.

b Ref. S30

c Ref. S31

d Ref. $\overline{\mathrm{S} 32}$

e Ref. S23

As mentioned previously, the effective defect volume in the QD model is given by $V_{\text {eff }}=4 \pi\left(\nu_{\mathrm{T}} a_{0}^{*}\right)^{3}$. Ridley [S29] proposed a form of the photoionization cross section that instead takes this volume to be an adjustable parameter. While useful for, e.g., fitting photoionization or photoluminescence spectra, this further reduces the predictive power of the model.

[S1] A. Alkauskas, Q. Yan, and C. G. Van de Walle, Phys. Rev. B 90, 075202 (2014).

[S2] K. D. Glinchuk, A. V. Prokhorovich, V. E. Rodionov, and V. I. Vovnenko, Phys. Status Solidi A 41, 659 (1977).

[S3] M. A. Reshchikov and H. Morkoç, J. Appl. Phys. 97, 061301 (2005).

[S4] M. A. Reshchikov, AIP Conf. Proc. 1583, 127 (2014)

[S5] M. Reshchikov, J. McNamara, M. Toporkov, V. Avrutin, H. Morkoç, A. Usikov, H. Helava, and Y. Makarov, Sci. Rep. 6, 37511 (2016).

[S6] E. W. Williams, Phys. Rev. 168, 922 (1968)

[S7] H. Queisser and C. Fuller, J. Appl. Phys. 37, 4895 (1966).

[S8] K. Glinchuk, A. Prokhorovich, and V. Vovnenko, Phys. Status Solidi A 34, 777 (1976).

[S9] F. Vorobkalo, K. Glinchuk, A. Prokhorovich, and G. John, Phys. Status Solidi A 15, 287 (1973).

[S10] A. Gutkin, M. Reshchikov, and V. Sedov, Semiconductors 34, 1151 (2000).

[S11] A. Gutkin, M. Reshchikov, and V. Sosnovskii, Semicond. Sci. Technol. 9, 2247 (1994). 
[S12] K. Wuyts, G. Langouche, M. Van Rossum, and R. Silverans, Phys. Rev. B 45, 6297 (1992).

[S13] J. Gebauer, E. Weber, N. Jäger, K. Urban, and P. Ebert, Appl. Phys. Lett. 82, 2059 (2003).

[S14] G. Baraff and M. Schlüter, Phys. Rev. Lett. 55, 1327 (1985).

[S15] J. L. Lyons, A. Janotti, and C. G. Van de Walle, Appl. Phys. Lett. 97, 152108 (2010).

[S16] A. Alkauskas, J. L. Lyons, D. Steiauf, and C. G. Van de Walle, Phys. Rev. Lett. 109, 267401 (2012)

[S17] T. Ogino and M. Aoki, Jap. J. Appl. Phys. 19, 2395 (1980).

[S18] C. Seager, D. Tallant, J. Yu, and W. Götz, J. Lumin. 106, 115 (2004).

[S19] J. L. Lyons, A. Janotti, and C. G. Van de Walle, Phys. Rev. B 89, 035204 (2014).

[S20] A. Gutkin, M. Reshchikov, and V. Sedov, Semiconductors 31, 908 (1997).

[S21] J. P. Perdew, K. Burke, and M. Ernzerhof, Phys. Rev. Lett. 77, 3865 (1996).

[S22] J. Heyd, G. E. Scuseria, and M. Ernzerhof, J. Chem. Phys. 124, 219906 (2006)

[S23] C. E. Dreyer, A. Janotti, and C. G. Van de Walle, Appl. Phys. Lett. 102, 142105 (2013).

[S24] Q. Yan, E. Kioupakis, D. Jena, and C. G. Van de Walle, Phys. Rev. B 90, 121201 (2014)

[S25] J. P. Perdew, M. Ernzerhof, and K. Burke, J. Chem. Phys. 105, 9982 (1996).

[S26] C. Adamo and V. Barone, J. Chem. Phys. 110, 6158 (1999)

[S27] H. B. Bebb and R. A. Chapman, in Proc. 3rd Photoconductivity Conf., edited by E. M. Pell (Pergamon, Oxford, 1971) p. 245.

[S28] H. B. Bebb, Phys. Rev. 185, 1116 (1969).

[S29] B. K. Ridley, Quantum Processes in Semiconductors (Oxford University Press, 2013).

[S30] O. Madelung, Semiconductors: Data handbook (Springer Science \& Business Media, 2012).

[S31] T. Skauli, P. S. Kuo, K. L. Vodopyanov, T. J. Pinguet, O. Levi, L. A. Eyres, J. S. Harris, M. M. Fejer, B. Gerard, L. Becouarn, and E. Lallier, J. Appl. Phys. 94, 6447 (2003).

[S32] T. Kawashima, H. Yoshikawa, S. Adachi, S. Fuke, and K. Ohtsuka, J. Appl. Phys. 82, 3528 (1997). 